\title{
On The Unification of Legal Rules in The European Union
}

\author{
Bertrand Crettez \\ Libre, Université de Franche-Comté \\ Régis Deloche \\ Libre, Université de Franche-Comté \\ April 2005
}




\begin{abstract}
${ }^{1}$
Unification of legal rules in Europe in not a new phenomenon. However, nowadays, Europe is still an area with many different jurisdictions. This paper studies the process of unification of legal rules in the European Union within a non-cooperative game-theoretical framework. This paper contributes to the understanding of the process by concentrating on the role of the European Commission.

In the law-and-economics literature, it is argued that national legal rules will converge more or less spontaneously through the works of legislators and judges. But legal convergence in the European Union is not necessary: preferences toward legal rules differ across nation-states; substituting a legal sytem for another is costly; a coordination problem may arise.

We first study the interactions of two nation-states who chose non-cooperatively their legal rules. We shall argue that the action of the Commission is, at first sight, likely to eliminate the coordination problem (under certain conditions). Two factors are at work. First, the Commission has a certain expertise which enables it to propose new and perhaps more efficient rules (so that the choice of unification does not reduce to select a particular nation-state legal system). Second, the Commission may use a system of fines that induce nation-states to abide by its rules (once these rules are adopted by nation-states

Next, we refine our first model: the process of legal unification is viewed as a game where nation-states choose the game that they will play. They choose if they will try to reach an agreement without resorting to the actions of the Commission, or if they will play the game implicitely proposed by the Commission. This captures more precisely the action of the Commission, its "right of initiative", the publication of proposals in "green" or "white papers". In this second model, a coordination problem may arise.

J.E.L Classification Numbers: C72, K00.

KEY WORDS: Legal Uniformization, Law-and-Economics.
\end{abstract}

\footnotetext{
${ }^{1}$ The authors thank Bruno Deffains for helpful comments during a seminar given at CEDES, Université de Nancy 2.
} 


\section{Introduction}

Harmonizations of legal laws in Europe is by no way a new phenomenon. Outstanding examples are the Code Civil de Napoléon and the German Civil Code. The former took effect on 21 March 1804, and Napoléon imposed it in some of the countries that he occupied. The latter took effect on 1 january 1900 (see Backhaus (1998) who presents a very interesting account of the inception of the German civil law, and the importance of economic arguments during the process of creation). With numerous smaller revisions, both these great codifications have remained unchanged since. At the present moment, another process of unification of legal rules in the European Union is at work. This paper contributes to the understanding of the process by concentrating on the role of the European Commission.

There is a well-known argument that explains the convergence of national legal rules. It is especially found in the law-and-economics literature where it is argued that national legal rules will converge spontaneously in order to implement an efficient allocation of scare resources (see, e.g. the papers in Marciano and Josselin (2002) and notably Smits (2002), Mattei (1994), Ogus (1999), Garoupa and Ogus (2003)). To put it in a nutshell, convergence will be achieved through the works of legislators, judges and arbitrators, who will choose efficient legal rules.

However, convergence is not necessary. First, preferences toward legal rules may differ across nation-states. Second, even if preferences are not too different, substituting a legal system for another is costly; the cost may well be larger than the benefits (typically due to increases in international transactions). This fact is analyzed in a recent paper by Herings and Kanning (2002). These authors raise also a third problem, which is a coordination problem. They indeed investigate the issue of convergence of legal rules (in fact commercial laws) by using a non-cooperative game between two nation-states.

They show that it is well possible that the coordination game has many solutions. That is, there are several instances where, if a country chooses a legal system, it is in the interest of other countries to choose this very legal system. As the authors put it, "in the absence of any coordination, it is far from obvious that nation-states will ever succeed in selecting the very same commercial laws". Moreover, Herings and Kanning argue that this coordination will be difficult since "the gains to be reaped by adherence to the same commercial laws may not necessarily be distributed equally among the nation-states". They indicate as a result, that "a nation-state that is better to spur economic growth than all other nation-states will be 
able to influence the outcome of any coordination of decisions between nation-states in a profound way".

The purpose of this paper is to evaluate the relevance of the analysis of Herings and Kanning in the European Union. The analysis of these authors complements our understanding of legal convergence (it gives new insight, which contradict in part the traditional view of the law-and-economics literature). The law-and-economics literature, which relies on spontaneous mechanisms, is perhaps not well adapted to study legal convergence in the European Union. In this Union, the European Commission has a key role in the process of unification. Indeed, the Commision has the "right of initiative", it is responsible for drawing up new proposals. So, we shall concentrate on the consequences of the existence of the European Commission on the coordination problem.

In the next section, we shall present a model of the action of the European Commission with regard to the convergence of legal rules (it is an extension of the model used by Herings and Kanning). We shall argue that the action of the Commission is, at first sight, likely to eliminate the coordination problem (under certain conditions). Two factors are at work. First, the Commission has a certain expertise which enables it to propose new and perhaps more efficient rules (so that the choice of unification does not reduce to select a particular nation-state legal system). Second, the Commission may use a system of fines that induce nation-states to abide by its rules (once these rules are adopted by nation-states ${ }^{2}$ ).

In section 3, we shall refine our previous argument. We shall show that the coordination problem may exist when we enrich the decision process faced by nation-states. Indeed, these nation-states may not endorse a proposition of the Commission and they can try to reach an agreement without resorting to the action of the latter.

In section 4, we shall address the question of coordination. This coordination problem is real, and apparently, no usual refinements of Nash Equilibria allow us to eliminate the coordination problem (although there exists a forward induction argument which can in part do the job). This was already noted by Herings and Kanning (who addressed the coordination problem using the view point of Harsanyi and Selten (1988)). We also show that there is a way out of this problem if the Commission is allowed to use a special subsidy (which has the advantage that, in equilibrium, it will never have to be paid off).

\footnotetext{
${ }^{2}$ The fact that a rule is adopted by nation-states does not imply that it will be implemented : the existence of fines remind us of these fact.
} 


\section{A Simple Model of Legal Convergence in the European Union}

Unification of legal rules within the European Union involves many players and many strategies. However, following Herings and Kanning (2002), we shall simplify the analysis by using two-players games with finite strategy sets. The two players are two nation-states, which choose non cooperatively their legal rules.

In the first subsection, we shall recall the setup of Herings and Kanning. Each nation-state can either stick to its own legal rules or choose to switch to legal rules of the other nation-state.

In the second subsection we shall present a version of the model of Herings and Kanning which, in our view, is better suited to analyze the process of legal unification in the European Union (Herings and Kanning were indeed particularly interested in the unification of commercial laws). Each nation-state faces now three alternatives. The first two options are those which are considered by Herings and Kanning. But we also allow nationstates to switch to a new legal rule which is proposed by the European Commission.

\subsection{The setup of Herings and Kanning}

As was said above, there are two nation-states, $A$ and $B$. The preferences of a nation-state are those of a representative household. Let $\pi_{P}(s)$ be the associated payoff to the state $P, P=A, B$, when the nation-states choose the strategy profile $s=\left(s_{A}, s_{B}\right)$ (where $s_{A}$ is the pure strategy chosen by State $A$, while $s_{B}$ is the pure stragegy chosen by State $B$ ). The strategy set $S_{P}$ of state $P$ is simply $\{A, B\}$ and we let $S$ be equal to the cartesian product $S=S_{A} \times S_{B}$.

If the legal rules of State $P$ are adopted in both countries, the representative household of $P$ receives a payoff $\pi_{P}(P, P)=B_{P}^{P}$ and the representative household of the other State $Q$ receives a payoff $\pi_{Q}(P, P)=B_{P}^{Q}-C_{P}^{Q}$. The non-negative term $B_{P}^{P}$ (resp. $B_{P}^{Q}$ ) denotes the discounted benefits for $P$ (resp. $Q$ ) which arise possibly because of the extra cross border trade and commerce generated by $Q$ in switching to $P$. The (non-negative) term $C_{P}^{Q}$ denotes the discounted extra transaction costs incurred by $Q$ in switching to $P$.

The strategic form of the game at hand, in which there is a significant mixture of conflict and coincidence of interest between players, is shown 
in table 1.

Nation-State $B$

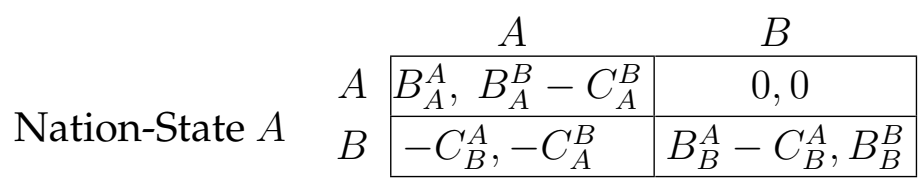

Table 1

In such situation, three basic game-theoretical structures can arise.

First, it may turn out that extra costs incurred by every nation-state of switching to the legal rules of any other nation-states exceed the extra benefits obtained: $B_{B}^{A}-C_{B}^{A}<0, B_{A}^{B}-C_{A}^{B}<0$. In such a case, $(A, B)$ is the unique Nash equilibrium of the game: adherence to one's legal rules is the single (dominant) optimal choice for each nation-state, no matter what the other nation-state does.

Secondly, it may turn out that there is only one nation-state for which the extra costs incurred in switching to the legal rules of the other nation-state exceed the extra benefits obtained. When $B_{B}^{A}-C_{B}^{A}<0$ and $B_{A}^{B}-C_{A}^{B}>0$, $(A, A)$ is the unique Nash equilibrium of the game: country $A$ will never switch to $B$ so that $A$ will be placed into a uniform legal rule.

Lastly, it may turn out that for all nation-state the extra costs incurred in switching to the legal rule of the other nation-states do not exceed the extra-benefit obtained. This corresponds to a coordination game: there are two Nash equilibria (in pure strategies): $(A, A)$ and $(B, B)$.

Note that when $B_{A}^{A}>B_{B}^{A}-C_{B}^{A}>0$ and $B_{A}^{B}-C_{A}^{B}>B_{B}^{B},(A, A)$ Paretodominates $(B, B)$. A symmetric case arises of course with $(B, B)$. On the other hand, when $B_{A}^{A}>B_{B}^{A}-C_{B}^{A}>0$ and $B_{B}^{B}>B_{A}^{B}-C_{A}^{B}>0$, or when $B_{B}^{A}-C_{B}^{A}>B_{A}^{A}$ and $B_{A}^{B}-C_{A}^{B}>B_{B}^{B}$, one cannot rank the Nash equilibria.

\subsection{Extension of the setup of Herings and Kanning}

As we have already mentioned, the preceding framework is a useful tool in order to analyze the unification of legal rules across nation-states insofar as it pertains to decentralized actions. There is no doubt that some legal rules, especially when they are essentially privately supplied, for instance through the process of arbitrage, may be seen as the outcome of a twoplayer non-cooperative game. 
Still, as far as the unification of legal rules in the European Union is concerned, and even if one still assumes that each nation state acts non-cooperatively, it may be more relevant to add some specific features.

First of all, the legal rules that apply across European nation-states are mostly proposed by the European Commission. We shall pay attention to this aspect in the next section. For the time being, it is sufficient to notice that the new rules proposed by the Commission are not necessary identical to the rules of one nation-state. Hence, the choice left to nation-states is not restricted to, say, the two national legal rules. The nation-states can also choose the legal rules proposed by the Commission. Taking this fact into account leads to a change in the payoff matrix which is now given in table 2, where $H$ denotes the action of switching to the legal rules proposed by the Commission.

Nation-State $B$

\begin{tabular}{|c|c|c|c|c|}
\hline & & $A$ & $B$ & $\mathrm{H}$ \\
\hline & $A$ & $B_{A}^{A}, B_{A}^{B}-C_{A}^{B}$ & 0,0 & $0,-C_{H}^{B}$ \\
\hline Nation-State $A$ & $B$ & $-C_{B}^{A},-C_{A}^{B}$ & $B_{B}^{A}-C_{B}^{A}, B_{B}^{B}$ & $-C_{B}^{A},-C_{H}^{B}$ \\
\hline & $H$ & $-C_{H}^{A},-C_{A}^{B}$ & $-C_{H}^{A}, 0$ & $B_{H}^{A}-C_{H}^{A}, B_{H}^{B}-C_{H}^{B}$ \\
\hline
\end{tabular}

Table 2

Second, European rules proposed by the Commission, be they reglements or directives, and adopted by the nation-states, enjoy a special property. Indeed, if nation-states rely upon another rule, they may have to pay fines.

Hence the task of the Commission is twofold: first it is a kind of legal adviser, which seeks the best legal rules for the nation-states; second, it makes sure that European rules are endorsed (using fines if necessary). This last fact leads us to modify the payoff matrix as follows:

Nation-State $B$

\begin{tabular}{cc|c|c|c|}
\multicolumn{1}{c}{} & \multicolumn{1}{c}{$A$} & \multicolumn{2}{c}{$B$} & \multicolumn{1}{c}{$A$} \\
\cline { 3 - 5 } Nation-State $A$ & $B_{A}^{A}-\epsilon, B_{A}^{B}-C_{A}^{B}-\epsilon$ & $-\epsilon,-\epsilon$ & $-\epsilon,-C_{H}^{B}$ \\
\cline { 3 - 5 } & $B$ & $-C_{B}^{A}-\epsilon,-C_{A}^{B}-\epsilon$ & $B_{B}^{A}-C_{B}^{A}-\epsilon, B_{B}^{B}-\epsilon$ & $-C_{B}^{A}-\epsilon,-C_{H}^{B}$ \\
\cline { 3 - 5 } & $H$ & $-C_{H}^{A},-C_{A}^{B}-\epsilon$ & $-C_{H}^{A},-\epsilon$ & $B_{H}^{A}-C_{H}^{A}, B_{H}^{B}-C_{H}^{B}$ \\
\cline { 3 - 5 } & & &
\end{tabular}

Table 3 
The main changes between tables 2 and 3 are the epsilons which capture the fact that when a nation-state does not endorse European rules, it faces the risk of being charged with fines. We have assumed that fines paid by one nation-state are not rebated to the other nation-states ${ }^{3}$.

Let us now look at the game whose payoffs matrix is given by table 3 . An important fact is that the coordination problem may be more difficult now than it was when the payoffs matrix was given by table 2 . Indeed, there may be three equilibrium ways to coordinate legal systems, namely $(A, A),(B, B)$, and $(H, H)$. Only the last equilibrium results from a kind of outside option proposed by the Commission. However, the coordination problem could disappear if the fines are well defined. This is shown in the following proposition.

Proposition 1 Assume that $B_{H}^{P}-C_{H}^{P} \geq-\epsilon, P=A, B$, and

$$
\epsilon>\max \left\{\min \left\{B_{A}^{B}-C_{A}^{B}+C_{H}^{B}, B_{A}^{A}+C_{H}^{A}\right\}, \min \left\{B_{B}^{A}-C_{B}^{A}+C_{H}^{A}, B_{B}^{B}+C_{H}^{B}\right\}\right\}
$$

Then the unique Nash equilibria of the game whose payoff matrix is given by table 3 is $(H, H)$.

Proof. It is clear that the condition $B_{H}^{P}-C_{H}^{P} \geq-\epsilon, P=A, B$ is necessary and sufficient for $(H, H)$ to be a Nash equilibrium. Let us now show that under our assumption, it is impossible that $(A, A)$ and $(B, B)$ are Nash equilibria. Note that if nation-state $B$ chooses $A$, nation-state $A$ will choose $H$ if $B_{A}^{A}+C_{H}^{A}<\epsilon$. If, in turn, nation-state $A$ chooses $A$, nation-state $B$ will choose $H$ whenever $B_{A}^{B}-C_{A}^{B}+C_{H}^{B}<\epsilon$. Under our assumptions, at least one of the above condition is satisfied so that $(A, A)$ is not a Nash equilibrium. The same reasoning shows that $(B, B)$ is not a Nash equililbrium. The conclusion follows. Q.E.D.

The preceding result yields a third reason why the Commission is behind the process of the unification of Legal Rules in the European Union. Through the system of fines, the Commission may solve the coordination problem. As a result, the allocation of resources can be improved (especially if the equilibrium $(H, H)$ Pareto-dominates the other equilibria).

\footnotetext{
${ }^{3}$ We believe that this assumption is rather relevant. In fact, the amounts of the fines are not important per se. What seems deterrent for a state, is the loss of reputation, or image, that results when it is charged with fines. So the epsilons capture the loss of reputation and this is why they do not have to be rebatted.
} 


\section{A More Complex Model of The Convergence of Legal Rules in The European Union}

In our view, the process that lies behind the harmonization of European laws relies upon the action of the European commission.

The commission proposes legislation and is in part responsible for implementing the decisions of Parliament and the Council. More precisely, the commission has the "right of initiatives". It is responsible for drawing up new European legislation.

In principle, if the "subsidiarity principle" is satisfied, the commission drafts a proposal. This proposal is based on extensive consultations. The commission is interested in gathering information on the need for fartherreaching action. Usually, the Commission includes a non-exhaustive list of possible solutions (other solutions may be suggested by any interested party).

The proposals are often published in green papers. In some case, these papers provide an impetus for subsequent legislation. The white papers sometimes follow a green paper; they contain an official set of proposals in specific policy areas.

The proposals are not always adopted by nation-states (for instance, in the area of contract law, it is likely that the issue of harmonization will be left to the market (see Smits (2002)), see also the reluctance of France to agree on further immediate deregulation).

The Commission must also monitor the application of European laws. As was already said, the Commission may use fines; it may also refer an "infringement" to the Court of Justice.

In the preceding section, we have analyzed the interactions between nationstates when there exists a European law, and how the existence of fines insures that this law is applied.

As we mentioned above, the nation-states can always refuse the proposals of the Commission. In so doing, the interactions between nation-states are similar to those analyzed by Hering and Kenning (there is no European legal rules, there are no fines). It seems sufficient that a single nation-state disagrees in order to stop the process.

In order to analyze the harmonization process, it seems revelant to study the joint decisions of nation-states on the proposal of harmonization made by the Commission.

The preceding ideas lead to the following remarks. In our view, the process of legal unification is best viewed as a non-cooperative game where 
nation-states choose the game that they will play. To put it differently, nation-states choose non-cooperatively if they will play the game implicitly proposed by the commission, the payoffs of which are given by table 3 , or, more simply, the game studied by Henings and Kanning. This leads to the following game in extensive form.

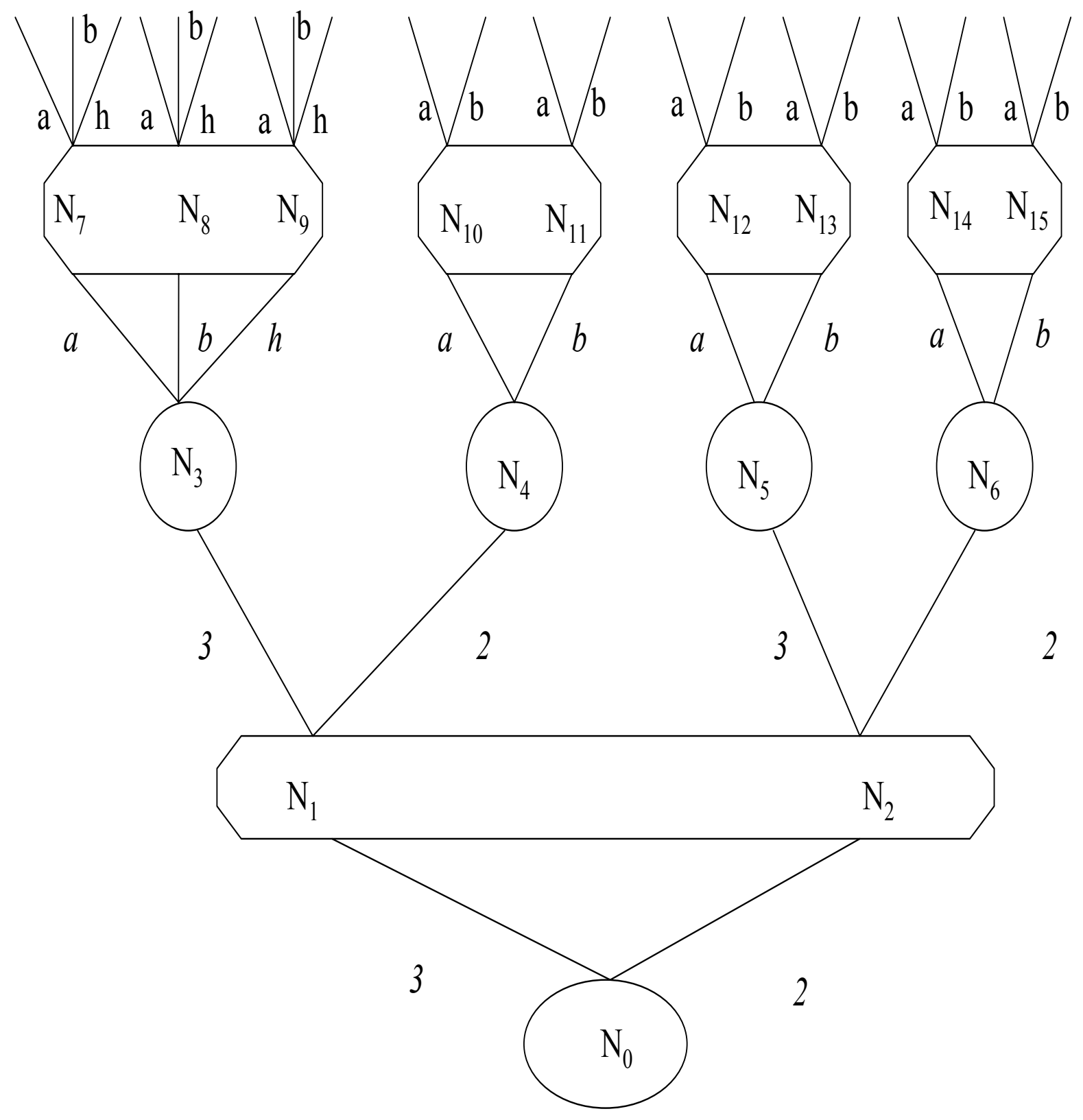

B

A

B

A 
Without loss of generality, we assume that the nation-state $A$ chooses first. At the initial node $N_{0}$, the alternative is either choosing the game with 3 possibilities of legal rules unification (implicitly proposed by the Commission), or the game where legal unification can only be achieved by adopting a national legal rule.

The immediate successor nodes of $N_{0}$ are in an information set since nation state $B$ does not know the move of nation state $A$. If node $N_{1}$ is reached, then two outcomes are possible. If nation-state $B$ chooses the game with three possible legal unifications, then, all the nodes which are successor of $N_{3}$ describe an extensive form of the simultaneous game whose payoff matrix is given by table 3 . On the other hand, if nation state $B$ chooses the game with two possible legal unifications, then the successors of $N_{4}$ describe an extensive form of the game whose payoff matrix is given by table 1 .

If node $N_{2}$ is reached, then, necessarily, only the game with two alternatives will be played. Accordingly, the nodes successor to $N_{5}$ and $N_{6}$ correspond to an extensive forme of the game associated to table 1.

The information sets $\left\{N_{7}, N_{8}, N_{9}\right\},\left\{N_{10}, N_{11}\right\},\left\{N_{12}, N_{13}\right\},\left\{N_{14}, N_{15}\right\}$ are the consequences of the assumption that nation-states move simultaneously. For instance, at node $N_{4}$, nation-state $A$ knows that the Commission's proposal is no endorsed, and chooses a legal rule; in the information set $\left\{N_{10}, N_{11}\right\}$, nation $B$ does not know the choice of nation-state $A$ and chooses its move.

Our task now is to analyze the Nash Equilibria of the game. The natural equilibrium concept to be used is the sub-game perfect Nash equilibrium. Recall that this concept requires that at an equilibrium, strategies are such that they generate a Nash equilibrium in every proper subgame.

The proper subgames of our game are the game itself, and the subgames that start respectively at nodes $N_{3}, N_{4}, N_{5}$ and $N_{6}$ (as we have seen above, the subgame that starts at node $N_{3}$ corresponds to the game with 3 options, while the others correspond to the game with only two options).

We shall not provide a very detailed analysis of the sub-game perfect equilibria of the game. In fact, we shall restrict ourselves to the case where the nation-states always choose to play $(H, H)$ once node $N_{3}$ is reached (this is indeed the case provided that the assumption of proposition 1 holds true). Also, we shall assume that there is a coordination problem (i.e. that both $(A, A),(B, B)$ are Nash equilibria of the game with 2 options). This allows us to adress the most difficult case, i.e., the case where there are a lot of equilibria). We shall also concentrate on the case where each nation-state 
prefers to switch to $(H, H)$ rather than adopting the other nation-state's legal rules. This is a particular case, but as will be seen below, even in this case there exists a coordination problem. Formally this amounts to assume that: for all $P, P=A, B, B_{H}^{P}-C_{H}^{P}>\max \left\{B_{P}^{P}, B_{Q}^{P}-C_{Q}^{P}\right\} \geq 0, Q \neq P$.

Sudying the Nash equilibria of our extensive form game involves specifying the correspondence bewteen information sets and the actions decided by the nation-states. This is done in table 4 .

\section{Strategy}

Profiles

\begin{tabular}{|c|c|c|c|c|}
\hline Information sets & type 1 & type 2 & type 3 & type 4 \\
\hline$N_{0}$ & 3 & 3 & 2 & 2 \\
\hline$\left\{N_{1}, N_{2}\right\}$ & 3 & 2 & 3 & 2 \\
\hline$N_{3}$ & $H$ & $H$ & $H$ & $H$ \\
\hline$N_{4}$ & $x$ & - & - & - \\
\hline$N_{5}$ & $y$ & - & - & - \\
\hline$N_{6}$ & $z$ & - & - & - \\
\hline$\left\{N_{7}, N_{8}, N_{9}\right\}$ & $H$ & $H$ & $H$ & $H$ \\
\hline$\left\{N_{10}, N_{11}\right\}$ & $x$ & - & - & - \\
\hline$\left\{N_{12}, N_{13}\right\}$ & $y$ & - & - & - \\
\hline$\left\{N_{14}, N_{15}\right\}$ & $z$ & - & - & - \\
\hline
\end{tabular}

Table 4

In this table the actions $(x, y, z)$ may be the following: $(A, A, A),(A, A, B)$, $(A, B, A),(B, A, A),(A, B, B),(B, A, B),(B, B, A)$ and $(B, B, B)$. The stragegy profiles are divided among four types. In the first type, nation-state will choose $(H, H)$. The other types will lead to different ways of coordinating legal rules.

Proposition 2 Assume that for all $P, P=A, B, B_{H}^{P}-C_{H}^{P}>\max \left\{B_{P}^{P}, B_{Q}^{P}-\right.$ $\left.C_{Q}^{P}\right\} \geq 0, Q \neq P$. Assume also that

$$
\epsilon>\max \left\{\min \left\{B_{A}^{B}-C_{A}^{B}+C_{H}^{B}, B_{A}^{A}+C_{H}^{A}\right\}, \min \left\{B_{B}^{A}-C_{B}^{A}+C_{H}^{A}, B_{B}^{B}+C_{H}^{B}\right\}\right\}
$$

Then, there does not exist subgame perfect Nash equilibria corresponding to strategy profiles of type 2 and 3. 
Proof. Let us prove that there is no subgame perfect equilibria of type 2. Clearly, if nation-state $A$ plays 3 , under our assumption and given proposition 1, nation-state $B$ should not play 2. As for the equilibria of type 3, observe that nation-state $A$ should not play 2 knowing that nation-statesstate $B$ plays 3. Q.E.D.

Let us now turn to equilibria corresponding to stragegy-profile of type 4 .

Proposition 3 Consider the equilibria associated to the strategy-profile of type 4. There always exists an equilibrium with $(x, y, z)=(P, P, P), P=A, B$. There exists an equilibrium with:

a) $(x, y, z)=(A, A, B)$ iff $B_{B}^{A}-C_{B}^{A} \geq B_{A}^{A}, B_{B}^{B} \geq B_{A}^{B}-C_{A}^{B}$;

b) $(x, y, z)=(A, B, A)$ iff $B_{A}^{B}-C_{A}^{B} \geq B_{B}^{B}$;

c) $(x, y, z)=(B, A, A)$ iff $B_{A}^{A} \geq B_{B}^{A}-C_{B}^{A}$;

d) $(x, y, z)=(A, B, B)$ iff $B_{B}^{A}-C_{B}^{A} \geq B_{A}^{A}$;

e) $(x, y, z)=(B, A, B)$ iff $B_{B}^{B} \geq B_{A}^{B}-C_{A}^{B}$;

f) $(x, y, z)=(B, B, A)$ iff $B_{A}^{A} \geq B_{B}^{A}-C_{B}^{A}, B_{A}^{B}-C_{A}^{B} \geq B_{B}^{B}$.

Proof. The proposition follows directly from the definition of a subgame perfect Nash equilibrum. Q.E.D.

Now, consider the equilibria associated to the strategy profile of type 1 . An argument similar to that of proposition 3 shows that there exists 8 equilibria where the strategy profile of type 1 is played.

There are then two facts that are worth to mention.

First, there are a multiplicity of the strategy profiles that can be played in a subgame perfect equilibrium. In particular, under our assumptions, there are no a priori reasons why the first strategy profile, i.e. the one that leads to European Harmonization of laws $((H, H))$, should necessarily be played.

Even it one could devise an argument eliminating one of the two remaining strategy profiles, there are a multiplicity of subgame perfect equilibria corresponding to a given profile. This is especially clear with the first strategy profile. Though $(H, H)$ is the final outcome, it is supported by various choices of actions outside the equilibrium path.

We have then illustrated the fact, that even when $(H, H)$ is strictly prefered to alternative ways of harmonizing laws in Europe, it is by no means sure that it will be the outcome of the interactions of nation-states. As long as nation-states act non-cooperatively, there exists a potential coordination problem. There does not seem to exist a simple way out of this problem. 
However this problem should not be overstated. In our model, the negotiation process is not explicitely modelled (this is a rather difficult issue, on this, see, e.g., Kreps (1990), page 376). There are many reasons why harmonization of European laws may not progress. One is simply that nationstates do not gain to the harmonization process. What we have pointed at, is that, even if harmonization of European laws generates welfare gains for all nation-states, a coordination failure can prevent these nation-states from implementing it.

\section{Unification of Legal Rules in the European Union and the Coordination Problem}

In this section, we shall study the coordination problem that was seen above. We shall adress this problem using two different arguments. First of all, we shall briefly look for simple refinements of the Nash equilibrium concept that could allow us to eliminate equilibria associated to strategy profile of type 4 . Second, we shall show that using subsidies, the Commission can solve - in part - the coordination problem. What is interesting is that, in equilibrium, these subsidies do not have to be paid off.

\subsection{Refinements of Nash Equilibrium and the coordina- tion problem}

The coordination problem has already been studied by Herings and Kanning (2002). They rely upon the notion of risk dominance introduced by Harsanyi and Selten (1988) in order to deal with the multiplicity of equilibria.

We have not followed this idea. Instead, we have explored some refinements that are of more common use. Notice that the popular devices proposed by Kreps and his co-writers cannot be applied to our context since they are more suited for games where there is uncertainty with regard to the types of agents.

It can be shown that all equilibria are sequential, so, this refinement is not very useful.

There is a peculiar refinement concept, proposed by Fudenberg and Tirole (1991), page 464, which they attribute to van Damme:

A solution concept $S$ is consistent with forward induction in the class of generic two-person extensive forms if there is no equilib- 
rium in $S$ such that some player $i$, by deviating at a node along the equilibrium path, can ensure (with probability 1) that a proper subgame $\Gamma$ is reached where (according to $S$ ) all solutions but one give the player strictly less than the equilibrium, and where exactly one solution gives the player strictly more.

This is a very interesting solution concept. It applies almost nicely to our set up when we assume that the outcome associated to the stragegy profile of type 1 strictly dominates the oucomes corresponding to those of the strategy profile of type 4 (we assume that there are no equilibria corresponding to the other strategy profiles).

If there were a unique equilibrium with strategy profile of type 1 , we could eliminate straightforwardly the equilibria with stragy profile of type 4 . Indeed, recall that the whole game is a proper subgame. So, by deviating, i.e. choosing to play according to the strategy profile of type 1 , states $A$ and $B$ can ensure a strictly better gain than by sticking to the choices $(2,2)$.

However, one cannot conclude that the equilibria corresponding to the strategy profile of type 4 are not consistent. This is because, as was already mentionned, there are multiple equilibria corresponding to the strategy profile of type 1 (whereas uniqueness is required).

We are not aware of other simple refinements concepts. They are likely to be based on sophisticated arguments, which make them unsuited to our framework. This is why it is probably more wise to look for other devices.

\subsection{Solving the coordination problem using subsidies}

The preceding arguments do not seem to provide a totaly convincing way to solve the coordination problem. We shall propose a way-out of the coordination problem that does not rely upon refinements provided by game theory. It consists in introducing a subsidy which should be given to any nation-state that favored harmonization if the latter is not implemented.

This amounts to add a term $S$ to the gains of nation-state $A$ whenever node $N 4$ is reached; or the gains of nation-state $B$, if node $N 5$ is reached.

For the time being, we shall remain silent about the way the subsidy is financed.

Proposition 4 We assume that the assumptions of Proposition 1 hold true. Assume that $S>\left|B_{A}^{A}-B_{B}^{A}+C_{B}^{A}\right|$. Then, there only exist equilibria associated to the strategy profile of type 1 . 
Proof. It is sufficient to prove that nation-state $A$ will always choose the coordination way proposed by the Commission (i.e. chooses 3 ).

Consider the stragegy profiles of type 4 where $(x, y, z)=(P, P, P), P=$ $A, B$. For any $S>0$, nation-state $A$ should choose 3 since its gains after node $N 4$ is reached is $B_{A}^{A}+S$ (resp. $\left.B_{B}^{A}-C_{B}^{A}+S\right)$ instead of $B_{A}^{A}$ (resp. $B_{B}^{A}-$ $C_{A}^{B}$ ) if $P=A$ (resp. $P=B$ ). Using a similar argument, one eliminates the stratefy profile with $(x, y, z)=(A, B, A)$ and $(B, A, B)$. The other strategy profiles of type 4 correspond to $(x, y, z)$ where $x \neq z$. There are two cases, $x=A, z=B$ and $x=B, z=B$. In the first case, the associated stragegy profile is not an equilibrium if: $B_{A}^{A}+S \geq B_{B}^{A}-C_{B}^{A}$. In the second, the corresponding strategy profile is not an equilibrium if $B_{B}^{A}-C_{B}^{A}+S>B_{A}^{A}$. Hence, it is sufficient that $S>\left|B_{A}^{A}-B_{B}^{A}+C_{B}^{A}\right|$. Q.E.D.

We now address the issue of the financing of the subsidy. Assume that the assumptions of propositions 1 and 4 hold true and that $S>\mid B_{A}^{A}-B_{B}^{A}+$ $C_{B}^{A} \mid$. Hence, from propositions 1 and 4 , the harmonization organized by the Commission is always chosen by the nation-states. Then, in equilibrium the subsidy is never paid off and so, the financing issue is irrelevant.

\section{Conclusion}

This paper was devoted to a study of unification of legal rules in Europe. We have first studied the interactions of two nation-states who choose non-cooperatively their legal rules. The task of the Commission is twofold: it seeks the best legal rules for the nation-states, so that the choice of unification does not reduce to select a particular nation-state legal system; it monitors the application of European laws, using fines if necessary. We have provided conditions under which the coordination problem is eliminated.

Second, we have refined our first model: the process of legal unification is viewed as a game where nation-states choose the game that they will play. They choose if they will try to reach an agreement without resorting to the actions of the Commission, or if they will play the game implicitely proposed by the Commission.

We believe that this new model captures nicely the action of the Commission, its "right of initiative", the publication of proposal in "green" or "white papers". We have seen that in this second model, a coordination problem may arise.

As was argued in the paper, eliminating the coordination problem is not an easy task. From a game-theorical view point, there is apparently no 
easy argument that could refine the multiple equilibria. However, we have shown that using a subsidy could help eliminating the "bad" equilibria.

The legal unification process in Europe is by no means restricted to the sole action of the European Commission. There is no question that some sponatenous actions are at hand. Moreover, the decisions of the Court of Justice are also important. Studying the actions of the Court is a natural topic for further research. 


\section{References}

1. Backhaus J. G., 1998, "Harmonization of law in the European Union", in P. Newman, The New Palgrave Dictionary of Economics and the Law, Volume 2, 211-215, Macmillan, London.

2. Fudenberg D. and J. Tirole, 1991, Game Theory, The M.I.T. Press, Cambridge, Massachussets.

3. Garoupa, N. and A. Ogus, 2003, "A Strategic Interpretation of Legal Transplants", W.P.

4. Harsanyi John C. and Reinhard Selten, 1988, A general Theory of General Equilibrium Selection in Games The MIT Press, Cambridge, Massachussets

5. Herings, PJ.J. and A.J. Kanning, 2002, "Unifying Commercial Laws of nation-states, Coordination of Legal Systems and Economics Growth", Working Paper.

6. Kreps, D.M., 1990, A Course in Microeconomic Theory, HarvesterWheatsheaf.

7. Ogus, A., 1999, "Competition Between National Legal Systems: A Contribution of Economic Analysis to Comparative Law", ICLQ, 2, 48,410 .

8. Marciano, A. and J.M. Josselin, 2002, The Economics of Harmonizing European Law, Edward Elgar, Pub., Cheltenham, Northampton.

9. Mattei, U., 1994, "Efficiency in Legal Transplants: An Essay in Comparative Law and Economics", IRLE, 14, 10.

10. Smits, J., 2002, "How To Predict The Differences In Uniformity Between Different Areas Of a Future European Private Law ? An Evolutionary Approach", in Marciano and Josselin (2002), 50- 70.

11. Wagner, G., 2002, "The Economics of Harmonization: The Case of Contract Law", Common Market Law Review, 39, 995-1023. 